\title{
Pathological and radiological correlation in an autopsy case of combined pulmonary fibrosis and emphysema
}

This article was published in the following Dove Press journal:

International Journal of COPD

8 July 2015

Number of times this article has been viewed

\author{
Hiroki Karata' \\ Tomonori Tanaka' \\ Ryoko Egashira ${ }^{2}$ \\ Kazuhiro Tabata' \\ Kyoko Otani ${ }^{3}$ \\ Ryuji Hayashi ${ }^{4}$ \\ Takashi Hori ${ }^{5}$ \\ Junya Fukuoka' \\ 'Department of Pathology, Nagasaki \\ University Graduate School of \\ Biomedical Sciences, Nagasaki, Japan; \\ ${ }^{2}$ Department of Radiology, Faculty of \\ Medicine, Saga University, Saga, Japan; \\ ${ }^{3}$ Department of Diagnostic Pathology, \\ Kobe University Graduate School of \\ Medicine, Kobe, Japan; ${ }^{4}$ Department \\ of Internal Medicine, University of \\ Toyama, Faculty of Medicine, Toyama, \\ Japan; ${ }^{5}$ Laboratory of Pathology, \\ Toyama University Hospital, \\ Toyama, Japan
}

Correspondence: Junya Fukuoka Department of Pathology, Nagasaki University Graduate School of Biomedical Sciences, I-7-I, Sakamoto, Nagasaki 852-850I, Japan

$\mathrm{Tel}+81958197055$

Fax +8I 958197056

Emailfukuokaj@nagasaki-u.ac.jp

\begin{abstract}
We report an educational autopsy case of combined pulmonary fibrosis and emphysema. Radiological patterns of the upper lung were considered as mostly emphysema, but pathological observation revealed significant interstitial fibrosis of usual interstitial pneumonia as a major pathology. The patient eventually developed acute exacerbation of background interstitial pneumonia. Careful radiological and pathological correlation of the current case indicates that regions with distal acinar emphysema on computed tomography image may possess histologically marked dense fibrosis of lethal interstitial pneumonia.
\end{abstract}

Keywords: interstitial pneumonia, CPFE, AEF, smoking, CT

\section{Introduction}

Historically, pulmonary emphysema is a classic condition of obstructive disease while pulmonary fibrosis is a prototype of restrictive disease. These two conditions had been considered as diseases of different spectrums. However, coexistence of pulmonary emphysema and fibrosis has been reported increasingly during recent years, in which Cottin et al proposed a new concept of combined pulmonary fibrosis and emphysema (CPFE) as a distinctive condition of diffuse lung disease. ${ }^{1}$ Cases with such combined condition have been widely recognized, ${ }^{2,3}$ however, in the current American Thoracic Society/European Respiratory Society (ATS/ERS) statement of idiopathic interstitial pneumonias, CPFE is described as an example of coexisting patterns comprising a heterogeneous population of patients and a syndrome which does not achieve a consensual understanding in the societies. ${ }^{4}$

An increasing number of publications describe combination of emphysema and interstitial fibrosis as having frequent association with carcinomas and pulmonary hypertension..$^{5-8}$ Accurate recognition of background interstitial fibrosis may have high clinical importance considering the risk of lethal acute exacerbation. The biological relationship between emphysema and interstitial fibrosis is still uncertain and whether they are two different diseases simply associated with cigarette smoking or diseases of one spectrum. In that sense, studies of careful radiological and pathological correlation of combined emphysema and fibrosis cases are needed.

\section{Clinical history}

An 83-year old male with smoking history of 63 pack years was diagnosed with interstitial lung disease by chest radiograph at 76 years of age. Before presentation he had also recently been diagnosed with rheumatoid arthritis based on 2010 Rheumatoid Arthritis Classification and had been on low dose of prednisolone $(5 \mathrm{mg} / \mathrm{day}) .{ }^{9}$ 
He then presented with respiratory symptoms after 2 weeks and pulmonary medicine specialists were consulted. He was treated with antibiotics under clinical diagnosis of infectious pneumonia, but did not respond well to treatment and further presented with right chest pain and fatigue a week later. At that time, he also developed renal dysfunction. Due to the serum elevation of myeloperoxidase anti-neutrophil cytoplasmic antibody (MPO-ANCA), the patient was suspected to have ANCA-related glomerular nephritis and was administered high dose methylprednisolone (500 mg/day). Computed tomography (CT) images were taken at the time of admission revealing a right lower lobe mass, which was biopsied and confirmed to be pulmonary adenocarcinoma. Due to the advanced stage of the carcinoma and patient's poor performance status, best supportive care was selected. Two weeks after admission, pulmonary dysfunction rapidly progressed to respiratory failure, resulting in his passing 1 month after admission. $\mathrm{His} \mathrm{FiO}_{2} / \mathrm{PaO}_{2}$ level at that time was $60 \mathrm{mmHg}$. Pulmonary function test was not performed due to the poor physical status of the patient. Serological test at the time of admission indicated the following abnormalities: CRP $16.56 \mathrm{mg} / \mathrm{dL}$; KL-6- 520.8 U/mL; MPO-ANCA$257 \mathrm{IU} / \mathrm{mL}$; antinuclear antibody $\times 160$.

\section{Radiological findings}

CT images taken at the time of admission showed paraseptal and centriacinar emphysema with mild reticulation predominantly in both upper lobes (Figure 1A). Fine and coarse reticulation with mild bronchiectasis and honeycomb-like cystic airspaces were also seen with basal and peripheral predominance (Figure 1B, C), which were originally considered to be a combination of upper-zone simple emphysema and lowerzone pulmonary fibrosis. At the top of the right lower lobe, a lobulated solid mass of adenocarcinoma was seen (Figure 1B). The marginal zone of emphysematous areas was relatively clear and irregular compared to "simple" emphysema (Figure 1A). Some areas of emphysema were admixed with reticulation, and fine reticulations and limited ground glass opacification were seen adjacent to the low attenuation areas.

\section{Pathological findings}

By gross pathology, subpleural cystic changes were obvious throughout craniocaudal levels, most prominent in the lower lungs. Remaining lung areas were yellow to brown in color with solid appearance (Figure 2).

Histologically, lower lungs indicated numerous cystic spaces corresponding to honeycomb cysts bilaterally, in which several cystic spaces were surrounded by dense fibrosis and filled with mucus. Most of these cysts were covered by the columnar bronchiolar type epithelia. Right lower lobe showed emphysematous lesions radiologically and macroscopically, but microscopic observation revealed marked interstitial fibrosis and smooth muscle hyperplasia (Figure 3). Patchy, peripheral-dominant dense fibrosis was observed. Fibroblastic foci were inconspicuous but found in the wall of honeycomb cysts. Right upper lobe appearing to be non-fibrotic tissue radiologically also demonstrates focal interstitial fibrosis and smooth muscle hyperplasia (Figure 4). Furthermore, left upper lobe indicates paraseptal emphysema with minimal reticulation in the radiological observation. However, it turned out to have remarkable fibrotic changes histologically corresponding to usual interstitial pneumonia (Figure 5).

\section{Discussion}

Histopathological analysis of the autopsied CPFE case revealed a surprising discordance between the impression of
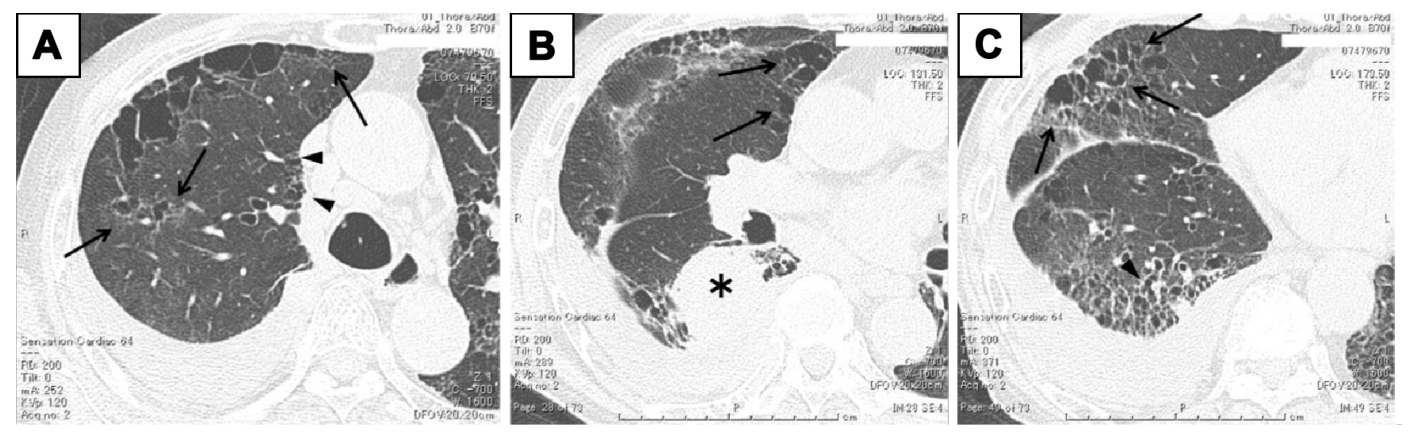

Figure I HRCT images of the case.

Notes: (A) HRCT image from right upper lobe: there are mainly paraseptal emphysematous changes in both upper lobes. Note the fine reticulations and limited ground glass opacification which are adjacent to the low attenuation areas (arrows). Some areas of emphysema are admixed with reticulation (arrowheads). (B, C) HRCT images from right middle to lower zone: there is both paraseptal and centrilobular emphysema. In the middle lobe, emphysema admixed with reticulation (C, arrows) is seen as well as in the upper zone (A). Compared to paraseptal emphysema without reticulation in the upper lobe, they show signs of interstitial abnormality (B, arrows). At the top of the right lobe, a lobulated solid mass is known lung cancer (B, asterisk). In the right lower lobe, there is fine and coarse reticulation with some cystic changes. The irregularly dilated airway, traction bronchiectasis (C, arrowhead), makes it possible to recognize the presence of pulmonary fibrosis (C).

Abbreviation: HRCT, high-resolution computed tomography. 


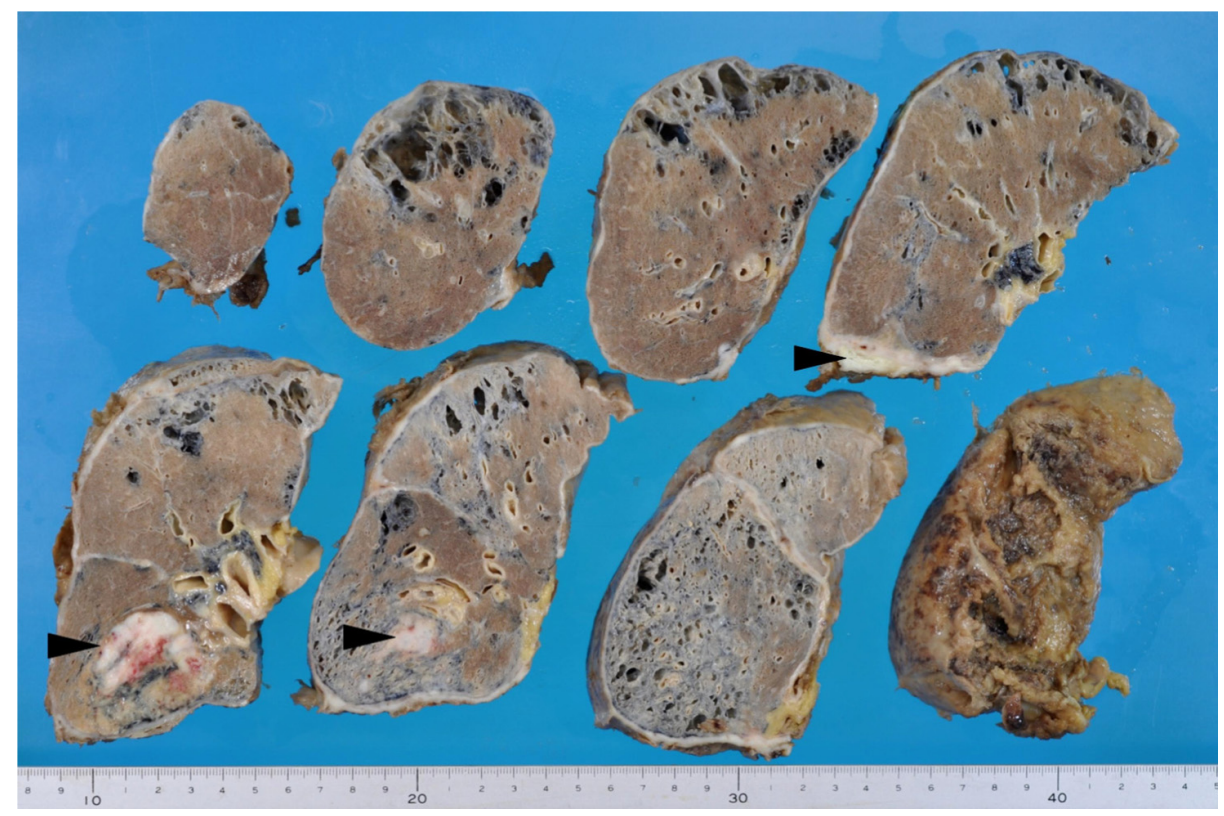

Figure 2 Gross appearance of the right lung.

Notes: Right lung was horizontally cut into eight slices. Top row is from upper lung areas showing emphysematous cystic spaces along with brown, normal-appearing area, while lower lung areas showed honeycomb changes. Arrowheads indicate pulmonary adenocarcinoma showing well circumscribed white, solid mass without remarkable necrosis.
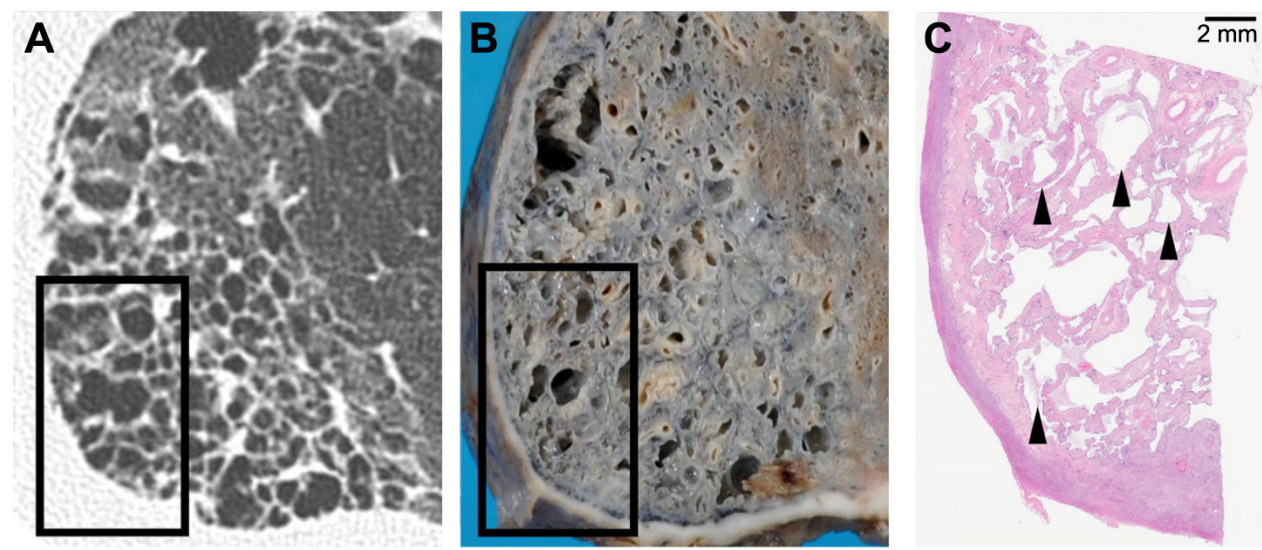

Figure 3 Computed tomography images and their corresponding histological images of right lower lobe.

Notes: (A) Computed tomography image shows coarse reticulation with honeycomb-like clustered cystic airspaces. Squared area corresponds to microscopic observation (C). (B) A gross picture of the same lesion. Squared area corresponds to microscopic observation (C). (C) Microscopic observation revealed cystic spaces and interstitial fibrosis. Arrowheads indicate areas corresponding to honeycomb cysts.
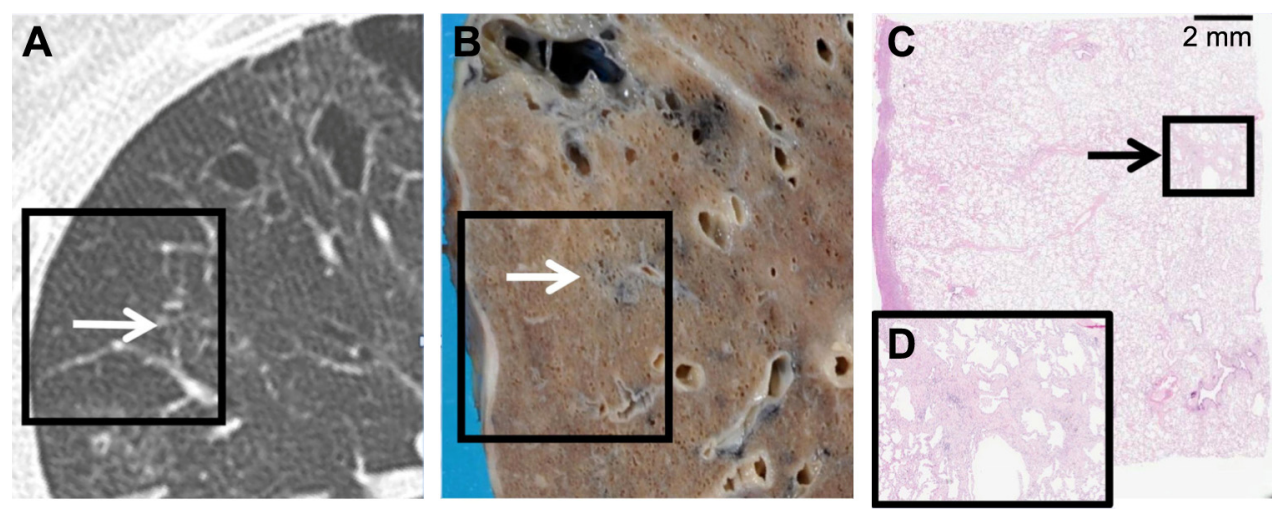

Figure 4 Computed tomography images and their corresponding histological images of right upper lobe.

Notes: (A) Computed tomography image shows subtle fine reticulation (arrow) which can be identified with careful observations. (B) A gross picture of the same area. (C) Microscopic observation of squared area in (A, B). (D) Close-up of the squared area in (C) revealed significant interstitial fibrosis. 
A

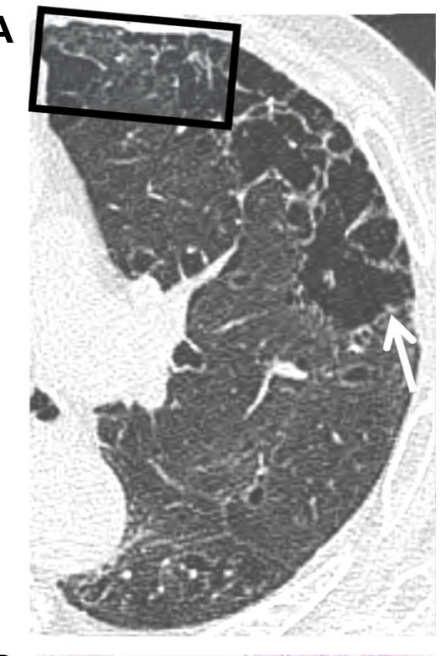

D

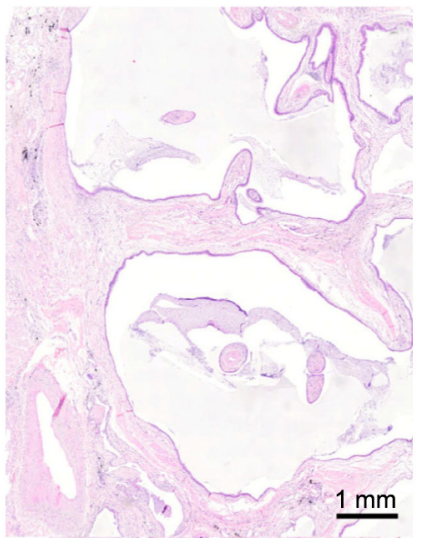

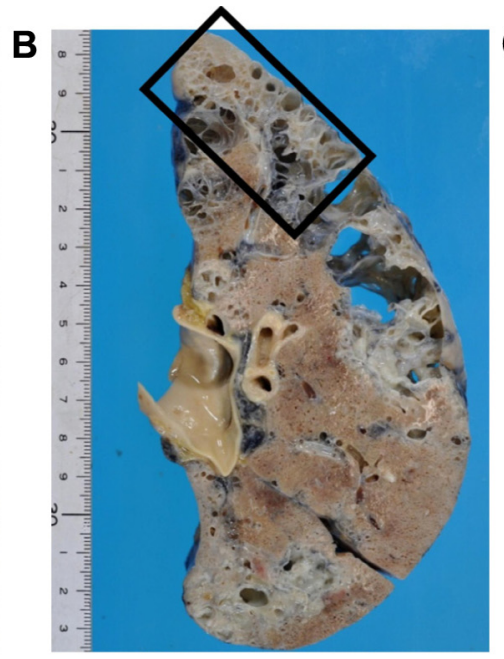

E

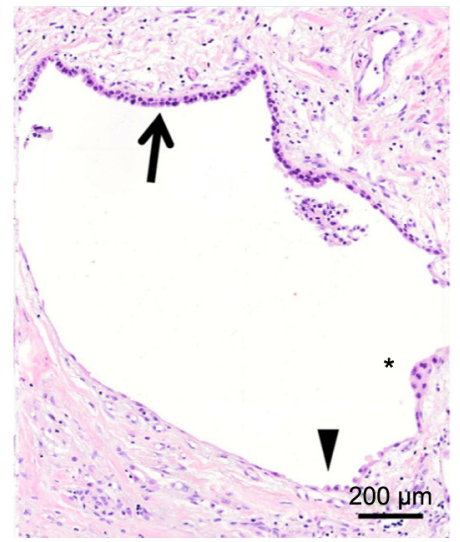

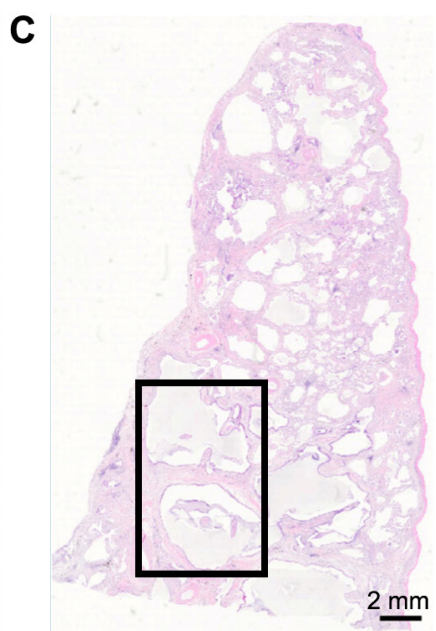

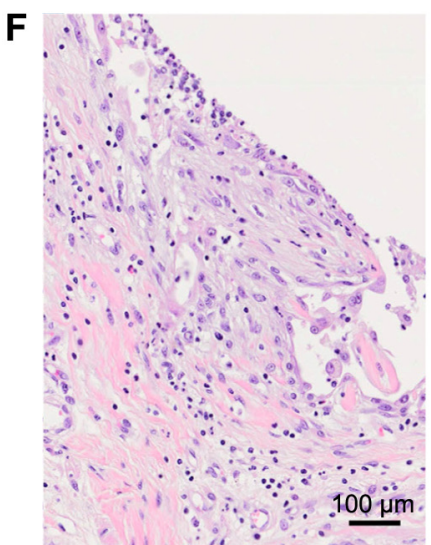

Figure 5 Computed tomography images and their corresponding histological images of left upper lobe.

Notes: (A) Computed tomography image shows mainly paraseptal and centriacinar emphysema. Note that the marginal zone of the emphysema tous area is clear and irregular than usual emphysema (arrow). (B) Gross appearance of the same area shows corresponding emphysematous-looking cystic spaces. (C) Histologically, significant fibrotic changes of usual interstitial pneumonia can be identified in the same area. (D) Higher magnification of image $\mathbf{C}$. Formation of honeycomb cysts are found in the upper lobe. (E) Honeycomb cysts are covered by mixture of bronchiolar epithelia (arrow), type II pneumocytes (arrowhead), and squamous metaplasia (asterisk). (F) Focal fibroblastic foci are found adjacent to dense fibrosis.

CT images and pathological findings. CT images of lower lung areas showed reticular shadow corresponding to pulmonary fibrosis. However, CT images of upper lung areas were originally considered to be simple emphysema. On the other hand, histological evaluation at autopsy revealed unexpected dense fibrosis of usual interstitial pneumonia (UIP) pattern both in the upper and lower lung areas.

This discrepancy is clinically important and should not be overlooked; in other words, pulmonologists and radiologists need to be informed of this as a warning. To our best knowledge, no case reports have been done on an acute exacerbation of diffuse alveolar damage due to emphysema alone. On the other hand, it is well known that a significant number of cases with pulmonary fibrosis, mainly UIP, accompanies acute respiratory failure leading to death.

As is described as characteristic histological patterns corresponding to the interstitial fibrosis seen in the smokers' lung, airspace enlargement with fibrosis (AEF) and smoking related interstitial fibrosis were reported. ${ }^{10,11}$ Although AEF and smoking related interstitial fibrosis are classified as subclinical conditions and clinically unimportant complications in the latest guidelines, ${ }^{4}$ two thirds of AEF cases are associated with UIP fibrosis, as Kawabata et al., report in their manuscript. ${ }^{11}$ Alternatively, majority of cases indicate the combination of AEF and UIP. Another critical problem of this context is that there is no consensus of how to diagnose and treat the patients with subtle interstitial fibrosis among "mostly emphysema" cases. Distinction between emphysema and interstitial fibrosis is difficult, and the clinical border of the two conditions seen within the same case is vague. Emphysema and pulmonary fibrosis were considered to be two different diseases, but the possibility that they are on common spectrum still remains. Recently, Stanley et al found mutations in TERT gene in emphysema patients. ${ }^{12}$ TERT gene mutation is also known as a mutation responsible for familial pulmonary fibrosis, and their 
report may explain why these two conditions are difficult to separate in certain cases.

Connective tissue disease, especially rheumatoid arthritis, is well known to be associated with pulmonary fibrosis. However, connective tissue disease-associated pulmonary fibrosis and idiopathic pulmonary fibrosis are often difficult to distinguish from one another. ${ }^{13}$ Similar situation applies to MPO-ANCA-related pulmonary fibrosis. ${ }^{14}$ However, there is a limited number of manuscripts describing the relationships between CPFE and autoimmune diseases including ANCA, thereby making the clear recognition of biological effect difficult, ${ }^{15-17}$ calling for further research in this field.

Cases with CPFE are known to have significantly higher risks of carcinoma association. ${ }^{8}$ This is an important factor to mention to pulmonologists, in order to urge careful observation of follow-up radiographs when patients present without any known carcinomas. As for cases that are already diagnosed with lung cancer, general surgical pathologists may focus only on staging and differentiating the histological types of cancer but not on observing background lung pathologies. Considering the risk of lethal acute exacerbation of pulmonary fibrosis, careful evaluation of radiographs to judge presence of background interstitial fibrosis is important. Some fibrosis mixed with emphysema may be subclinical and unimportant. However, pulmonary fibrosis generally possesses risks of acute exacerbation. Therefore, detection of fibrosis in the wall of emphysematous cysts as seen in the present case is critical.

\section{Conclusion}

We have examined an autopsy case showing emphysema and pulmonary fibrosis, in which the patient developed diffuse alveolar damage as an acute exacerbation. Radiological and pathological correlation of the current case indicates that simple emphysema on CT may possess characteristics of usual interstitial pneumonia histologically, which can later lead to lethal acute exacerbation.

\section{Disclosure}

The authors report no conflicts of interest in this work.

\section{References}

1. Cottin V, Nunes H, Brillet PY, et al. Combined pulmonary fibrosis and emphysema: a distinct underrecognised entity. Eur Respi J. 2005;26(4): 586-593.

2. Dodda VR, Salazar-Schicchi J, Spiro P. Combined pulmonary fibrosis and emphysema. Respir Care. 2012;57(3):467-470.

3. Takahashi M, Fukuoka J, Nitta N, et al. Imaging of pulmonary emphysema: a pictorial review. Int J Chron Obstruct Pulmon Dis. 2008;3(2): 193-204.

4. Travis WD, Costabel U, Hansell DM, et al. An official American Thoracic Society/European Respiratory Society statement: Update of the international multidisciplinary classification of the idiopathic interstitial pneumonias. Am J Respir Crit Care Med. 2013;188(6):733-748.

5. Usui K, Tanai C, Tanaka Y, Noda H, Ishihara T. The prevalence of pulmonary fibrosis combined with emphysema in patients with lung cancer. Respirology. 2011;16(2):326-331.

6. Sugino K, Ota H, Fukasawa Y, Uekusa T, Homma S. Pathological characteristics in idiopathic nonspecific interstitial pneumonia with emphysema and pulmonary hypertension. Respirol Case Rep. 2013;1(2):39-42.

7. Cottin V. Clinical case: Combined pulmonary fibrosis and emphysema with pulmonary hypertension - clinical management. BMC Res Notes. 2013;6 Suppl 1:S2.

8. Jankowich MD, Rounds SI. Combined pulmonary fibrosis and emphysema syndrome: a review. Chest. 2012;141(1):222-231.

9. Aletaha D, Neogi T, Silman AJ, et al. 2010 rheumatoid arthritis classification criteria: an American College of Rheumatology/European League Against Rheumatism collaborative initiative. Ann Rheum Dis. 2010; 69(9):1580-1588.

10. Katzenstein AL, Mukhopadhyay S, Zanardi C, Dexter E. Clinically occult interstitial fibrosis in smokers: classification and significance of a surprisingly common finding in lobectomy specimens. Hum Pathol. 2010;41(3):316-325.

11. Kawabata Y, Hoshi E, Murai K, et al. Smoking-related changes in the background lung of specimens resected for lung cancer: a semiquantitative study with correlation to postoperative course. Histopathology. 2008;53(6):707-714.

12. Stanley SE, Chen JJ, Podlevsky JD, et al. Telomerase mutations in smokers with severe emphysema. J Clin Invest. 2015;125(2):563-570.

13. Tabata K, Fukuoka J. Histopathologic features of usual interstitial pneumonia and related patterns: what is important for radiologists? Semin Ultrasound CT MR. 2014;35(1):2-11.

14. Tanaka T, Otani K, Egashira R, et al. Interstitial pneumonia associated with MPO-ANCA: clinicopathological features of nine patients. Respir Med. 2012;106(12):1765-1770.

15. Tzouvelekis A, Zacharis G, Oikonomou A, et al. Increased incidence of autoimmune markers in patients with combined pulmonary fibrosis and emphysema. BMC Pulm Med. 2013;13:31.

16. Cottin V, Nunes $\mathrm{H}$, Mouthon $\mathrm{L}$, et al. Combined pulmonary fibrosis and emphysema syndrome in connective tissue disease. Arthritis Rheum. 2011;63(1):295-304.

17. Homma S, Suzuki A, Sato K. Pulmonary involvement in ANCAassociated vasculitis from the view of the pulmonologist. Clin Exp Nephrol. 2013;17(5):667-671
International Journal of COPD

\section{Publish your work in this journal}

The International Journal of COPD is an international, peer-reviewed journal of therapeutics and pharmacology focusing on concise rapid reporting of clinical studies and reviews in COPD. Special focus is given to the pathophysiological processes underlying the disease, intervention programs, patient focused education, and self management protocols.

\section{Dovepress}

This journal is indexed on PubMed Central, MedLine and CAS. The manuscript management system is completely online and includes a very quick and fair peer-review system, which is all easy to use. Visit http://www.dovepress.com/testimonials.php to read real quotes from published authors. 